\title{
An SOA Framework for Web-based E-learning Systems - A case of Adult Learners
}

\author{
Eric Araka \\ School of Computing and Informatics \\ University of Nairobi \\ Nairobi, Kenya
}

\author{
Lawrence Muchemi \\ School of Computing and Informatics, \\ University of Nairobi \\ Nairobi, Kenya
}

\begin{abstract}
The existing e-learning design models for adult learners are mostly built on pedagogical principles and are more appropriate for younger learners. They may not support learning activities that may be suitable for adult learners. To make use of adult learners' principles, a dedicated learning model based on SOA, and intended to make learning process collaborative while allowing learners to use their experiences and self-directedness was developed. The SOA framework was able to deliver a remote service into a web-based LMS; in this case Moodle-based LMS so that it can be internally utilized by the adult learners in accessing learning materials from other systems as services. Analysis from the evaluation process was adequate enough to give a clear effect of using the SOA framework when compared to the initial survey carried out on the current e-learning systems which the learners used in their learning.
\end{abstract}

Keywords: eLearning, SOA, LMS, Andragogy, Adult learners

\section{INTRODUCTION}

The e-learning environments that are used to instruct adult learners are the same ones that are used to instruct children and do not obviously support diverse learning activities that may be suitable for adult learners. Pedagogy is not as useful in an adult learning environment because it does not utilize the learner's capabilities, experiences and adult learning characteristics [4] [5].

The wealth of real-life experiences that adult learners possess is a great resource that can be utilized for their learning. These experiences cannot be utilized in the current e-learning environments, which are only used to deliver information to the learners. Adult learners need an environment that can enable them to be involved in the construction of their own course content, giving then the freedom to make use of the experiences that they possess [10][11]. Bichelmeyer [3] argues that to this end e-learning for adult learners has been presented as though it only involves only one type of educational experience as it only provides learners with information they need with less emphasis on the learning process itself.

This research focuses on a design model of e-learning systems based on the theory of andragogy [14] (as an alternative to pedagogical model of instruction) to enhance learning for adult learners.

\section{LITERATURE REVIEW}

\subsection{Theory of Adult Learners}

Adult learners are those who perform roles associated with adults by one's culture e.g., workers, spouses, parents and perceive themselves to be responsible for their own lives [12]. Adults are experienced, self-directed and are independent thinkers in their learning and they seek to learn from and about their social and work environments and the roles they play there as opposed to young leaners. Learning by being guided by others is therefore unfitting in adult education environments [14].

According to Knowles [13] [14], andragogy rests on four crucial assumptions about adult learners and how they differ from child learners (1) their self-concept moves from dependence to self-direction, (2) their growing reservoir of experience begins to serve as a resource for learning, (3) their readiness to learn becomes oriented increasingly toward the developmental tasks of their social roles, and (4) they begin to want to apply what they have learned right away to life's real challenges. Adults' orientation towards learning shifts from one of subject centeredness to one of problem centeredness.

The review of andragogy theory indicates that adult learners are characterized by a great experience and self-directed learning [6]. E-learning systems for adult learners, therefore, should enable learners go through instructional materials delivered via the Web at their own pace with no or minimal interaction with an instructor and be involved in content creation. This theory also implies that adult learning should therefore emphasize on knowledge construction by learner actively exploring and discovering for.

Adult learners possess more life and domain specific knowledge, different motivations to learn, and more available resources than young learners [3]. Basing on this assumption, and adult learners' characteristic of self-directedness and experiences, the web-based system for e-learning described in this study allows adult learners to use this reservoir on experience and to be involved in the creation of content for learning. 


\subsection{Service Oriented Architecture \& Web} Services

Service Oriented Architecture (SOA) is a description of how different parts of the system interact and communicate to achieve a desired result. It is "an interconnected set of services which in its basic form is a message-based interaction between software agents, each accessible through standard interfaces and messaging protocol" [16]. SOA is implemented through the use of web services [17].

A number of SOA e-learning frameworks have been developed. These frameworks include the NSDL [21], OKIModel [21], IMS DRI [22] and as summarized by [15]. Scott (2003) also describes an SOA framework that integrates different systems of an institution that occupy vertical positions e.g. VLE, Library Management System and Student Records (MIS). The framework integrates the components of different systems in an institution in order to reduce replication/overlapping of functions.

"A web service is a software system designed to support interoperable machine-to-machine interaction over a network. It has an interface described in a machine-processable format called WSDL. Other systems interact with the web service in a manner prescribed by its description using SOAP messages, typically conveyed using HTTP with an XML serialization in conjunction with other Web-related standards."[1]

The importance of utilizing SOA in e-learning systems includes integration, interoperability, scalability, and reusability [17]. Interaction, knowledge building and collaboration features of e-Learning systems cannot be ignored and it is necessity that they be considered in the adoption of SOA in adult e-Learning systems. The attributes of web services that include reusability, composability, discoverability and loose coupling [16][17] extends the importance of employing SOA in e-learning systems to allow integration of different systems to communicate and utilize the services [18] they contain from each other.

There are a number of disparate systems that expose learning resources as web services and can be utilized within LMSs through the SOA framework. These web services include;

1. Repository of learning objects based on web services which in the context of this study refer to reusable content components for education and training.

2. Application Programming Interfaces: There are a number of programs written and stored on various applications as learning objects and may be accessed through APIs that are exposed as set of related web services that can be accessed through different protocols. This when brought close to students through the framework discussed in this study allows students to access written programs that are executed within the LMSs and enables students to solve complex programming exercises without having to install the API on their machines and even allow collaboration and grading during and after the programming exercises [15]
3. Evaluation engines. There are a number of evaluation engines that provide electronic quizzes, computerscored homework assignments, and practice exams.

The Prototype based on the SOA framework developed through this study is embedded in a Moodle-based LMS to allow for the access of the above services. The communication between the Service Oriented LMS (service consumer) and the other web based systems (service providers) is through message passing through the OAuth protocol which uses HTTP POST requests in its transport layer.

The SOA framework enhances adult learning while taking consideration of the adult learning principles. The framework helps adult learners employ the great reservoirs of experiences gained through professional work skills and knowledge in material construction as well as provision of services that will allow for opportunities for rehearsal, feedback, application, and transfer. The interaction and collaboration services are oriented in the current e-learning systems so as to be fit for adult learning.

\section{METHODOLOGY}

This study used an approach that involved the following procedure. First, an existing pedagogical-based eLearning system based at one of the leading public University in Kenya was investigated on the extent to which it implemented the adult learners' characteristics. Second, a survey (on a sample set of 228) to establish the motivational level of the adult students who were carrying their studies through e-learning was done. The results from the investigation and survey led to the development of a framework describing the principles of adult learners that need to be incorporated in an SOA framework. An andragogical-based e-learning framework that runs on an SOA model was developed from the adult learners' principles framework (Figure 1).

A prototype based on the andragogy based eLearning framework was then developed and was used in a simulated Moodle-based LMS. This prototype was used to validate the proposed SOA framework. A sample comprising of 58 students was used in the validation process.

\section{RESULTS, ANALYSIS \& DESIGN}

The findings from the approach described in the methodology are as follows.

\subsection{Findings from the investigation of the current e-learning environment}

The investigation of the eLearning system was important as it helped in the description of the design and the suitability of the e-learning environment to the adult learners who were engaged in this study. The main objective of the investigation was mainly to understand the design principles of the existing eLearning system and then provide the context for the design of an SOA based model for adult learners.

It was established that the e-learning environment used to instruct study participants who are adult learners is the same 
one used to instruct the undergraduate students. Grounded on the pedagogy principle, the e-learning system is used especially to deliver content or learning materials to the learners. The learning environment does not utilize the learner's capabilities, experiences and adult learning characteristics [4] [5].

\subsection{Findings from the study Survey}

The survey was carried out to establish the level of motivation and satisfaction on the adult learners who used the current eLearning system put in place for their learning. Part I of the survey collected background information regarding the survey participants. The respondents who participated in the survey are learners who are pursuing their postgraduate studies. Majority of the participants belong to the age group between $25-34$ and $35-44$ which represents $54 \%$ and $36 \%$ respectively.

From the analysis, $94.5 \%$ of the number who were involved in the research displayed the characteristics of an adult learner i.e. $79 \%$ were married, playing a role of a parent/spouse and had accomplished the undergraduate student period of four years of traditional college/university learning, $42 \%$ of whom are employed as working professionals. When asked for the reason for enrolling for the e-learning course only $4 \%$ indicated that their main reason was to receive a certificate, while $96 \%$ indicated that they enrolled in the e-learning course in order to develop and enhance their skills. This analysis agrees with what Knowles defined who an adult learner is [13].

Part II of the study explored the motivation/satisfaction of their learning process through e-learning. When asked about the frequency of their interaction with an instructor, a majority of those surveyed responded that they occasionally had interactions while they took the e-learning course. In particular, $12 \%$ of the respondents indicated that they never had such interactions and $76 \%$ of those surveyed responded that they occasionally or seldom interacted with an instructor.

As Holton [6] argues, 96\% of the respondents indicate that they like learning on their own pace. This reflects that any learning environment should give the adult learners intellectual freedom, experimentation and creativity. Sixty four (64) \% of those involved in the survey indicated that their achievements and previous experiences were not utilized in the learning process. Cook asserts to this as she argues that researchers with expertise in education and information communications technologies have not applied their findings to the adult learners. She continues to argue that this has resulted in teaching methods and strategies that are ineffective in teaching and instructing the adult learners. Of the adults who participated in this study $81 \%$ responded that they prefer interaction and collaboration with other students, as noted in the following comment by a participant who was a full-time working professional: "Free interaction with other students and instructors and sufficient e-learning material provided"
Adult learners possess characteristics that should be incorporated in their training. Experience, a great resource that can be tapped in the learning process and selfdirectedness, through which adult learners can participate in the learning process. These are special components in adult learning process which allow the learners pick and choose what they are only interested with.

From the survey conducted in this study it is clear that the current design models are not suited for adult learners. The same environment is used for both andragogy and pedagogy classes.

\section{Figure 1: Principles of adult learners implemented by the SOA Framework}

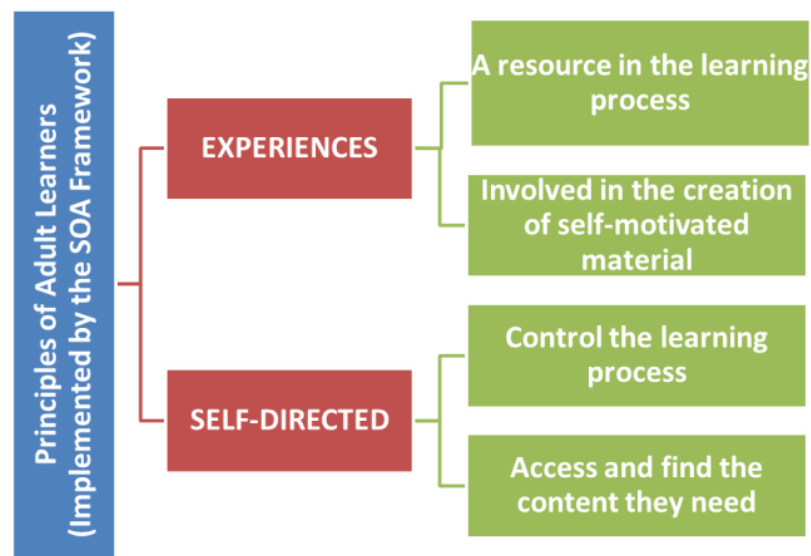

\subsection{The SOA Framework Design \\ 4.3.1 Context diagram of the SOA based integrating framework}

The SOA framework enables the Moodle Course students while logged into the LMS to connect over to other remote tool, blog or another LMS and be automatically authenticated and allowing them proceed to use their experience in selecting the content they want in the Moodle course(s).

Figure 2: Context diagram of the SOA based integrating framework

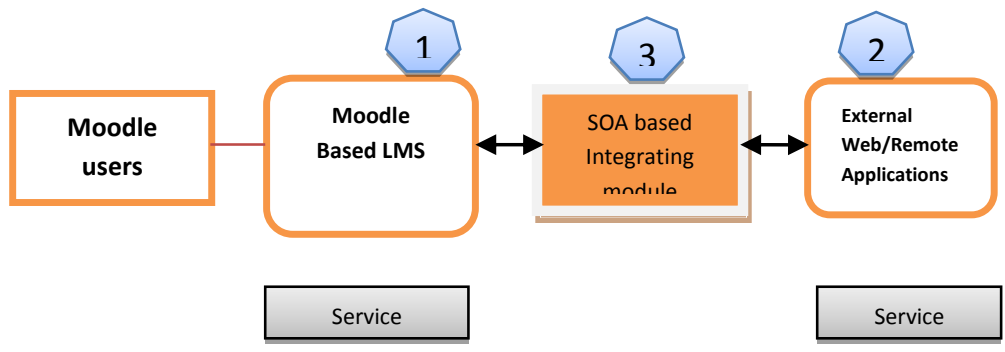

The architecture comprises of three parts: Service Consumer - Moodle LMS, acting as the service consumer, and containing the users of the e-learning management systems; External Web/Remote Application, acting as the service provider, it stores the remote content to be accessed by users of the LMS system upon clinking the Launch URL of the LTI integrator; SOA based integrating module, is the application 
at the core of this architecture which provides an API between the service consumer (Moodle LMS) and service provider (remote application). The SOA based integrating module is implemented through the use of IMS specification standards and allows embedding of the remote system in the LMS by assigning information in the launch process such as launch URL, shared key and the secret.

\subsubsection{The SOA Framework Architecture}

The SOA Framework provides a way for the service consumer (Moodle LMS) to send a user to another system i.e. service provider (this is the service that integrates with the LMS). It allows the user to be authenticated and allow access to a specific course when the service provider renders the content.

The service provider and service consumer communicates through the use of a consumer key and a shared secret which allow any message to be passed between the two systems. The messages are signed using the OAuth (soap uses this protocol over the transport layer) protocol for secure API authorization.

\section{Figure 3: The SOA Framework Architecture}

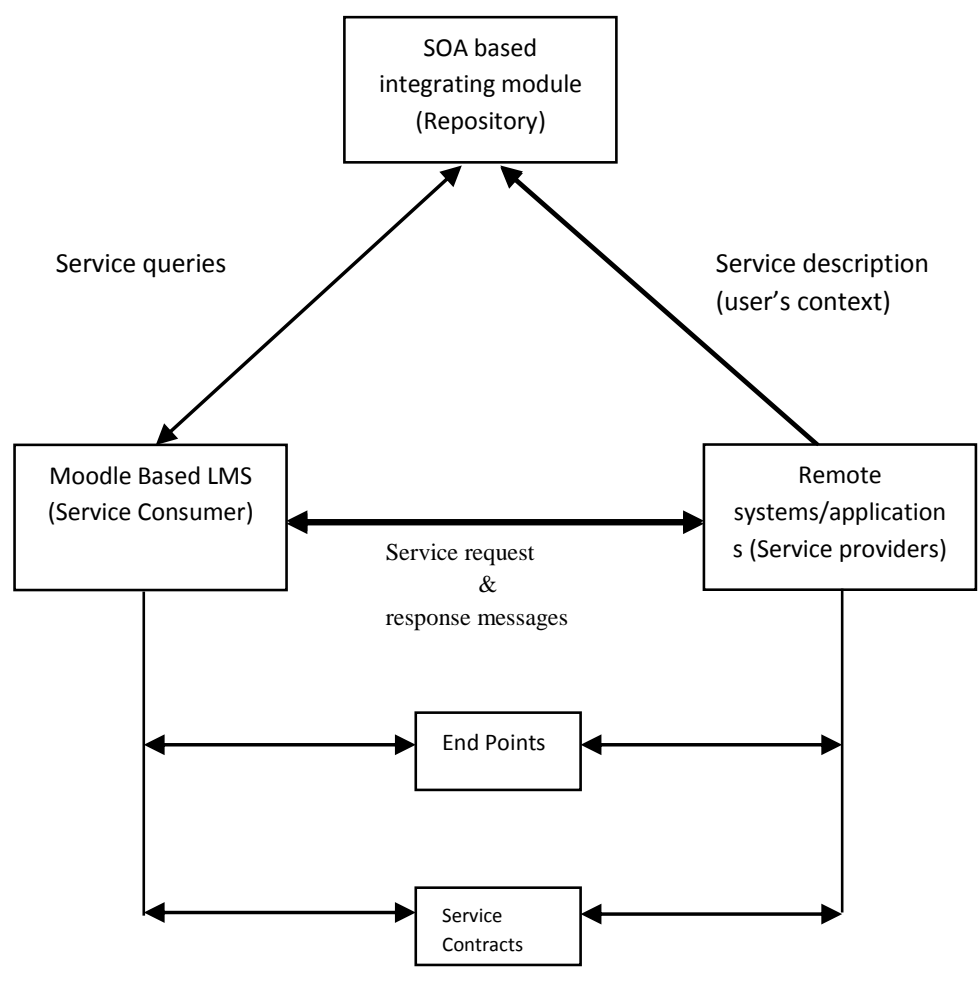

The endpoints provides communication to the service providers and the context for the connection, that is, what service has been requested, where to locate it and its final presentation on the service consumers browser. This is achieved through a graphical user interface which provides for configuration module where details about point-to-point connection between LMS and remote applications are entered. Here, the service consumer which is the LMS knows the endpoint (as configured) and send request is launched when the user clicks on the link(s) provided within the course.

The service contracts in this framework define the relationship between the provider and the consumer, that is, what the service provider will give to the consumer. The contract actually defines what functionality the provider provides to the LMS, what data it will return and in what form. The agreement between the LMS and service providers are entered into when the administrator fills out a form that typically provides the details governing the two interacting parties detailing what happens after the connection established after authentication.

\subsubsection{Messages and Communication between the SOA components}

The SOA Framework provides a way for the service consumer (Moodle LMS) to send a user to another system i.e. service provider (this is the service that integrates with the LMS). It allows the user to be authenticated and allow access to a specific course when the service provider renders the content. The service provider and service consumer communicates through the use of a consumer key and a shared secret which allow any message to be passed between the two systems. The messages are signed using the OAuth (soap uses this protocol over the transport layer) protocol for secure API authorization.

\subsection{Principles of Adult Learners implemented by the SOA framework}

This framework addresses the principles of adult learners as cited in Figure 1 as follows:

\subsubsection{Experience}

a. Resource in the learning process \& Creation of self-motivated learning material

The experience that adult learners possess is a great resource in the learning process. The design model, based on SOA, is intended to make eLearning process collaborative while allowing the learners to use their experiences in publishing articles within an e-learning system and to allow them share what they learn. The published articles are subjected to thoughtful contributions from other faculty groups; informalcompetency groups i.e. those who possess similar levels of knowledge and experience. As identified by Rodrigues [19] blogging, the method adopted by this research, leads to transformative learning which is based on learners' experiences and encourages reflection and free thinking leading improvement of knowledge. The combination of the contributions from the learners leads to material that one can use to refer to a specific topic of learning as contributed by different adult learners in their professional fields.

\subsubsection{Self-Directedness}

\section{a. Control the learning process}

The SOA framework when implemented on eLearning systems for adult learners, enables learners go through 
instructional materials delivered via the Web at their own pace with no or minimal interaction with an instructor.

\section{b. Access and find the content they need}

This is reflected in the SOA framework by providing to learners an e-learning environment that gives the adult learners intellectual freedom, experimentation and creativity. The different sources of learning materials exposed to the learners through the design model enables them access and find the content they need. This is in contrary to what the current pedagogical models provide.

\section{VALIDATION OF THE SOA FRAMEWORK}

The validation process was carried out on the prototype that was developed and simulated on the Moodle-based LMS. The purpose of the validation was used to establish whether the adult learners' characteristics identified in this study were incorporated in SOA framework. These included;

a) Use of adult learners experience in the enhancement of learning process and creation of self-motivated learning materials.

b) Self-directedness of adult learners so that;

i. They can control the learning process.

ii. They can access and find the content they need.

\subsection{Procedure used for evaluation}

The validation process involved the following procedure. First, the prototype was made available to the learners for inspection using Abstract Tasks (AT) approach. Abstract Tasks (AT) inspection method describes activities to be performed during the inspection and captures usability experience while identifying the application features on which it is important to focus inspection and describes the actions the inspector should perform during the evaluation [2]. AT inspection aims at allowing inspectors who may not have a wide experience in evaluating e-learning systems to perform accurate evaluations by performing specific tasks. A survey (on a sample size of 58) to establish increment on the motivational level compared to the initial survey (carried on the pedagogical based e-learning system) was then carried out. Secondly, system logs/views were collected from the prototype to measure;

i. $\quad$ The extent which learners employed the use of their experiences in the creation of self-motivated learning materials through collaboration amongst learners. The selfmotivated learning materials were created through the archived ideas exchanged by the learners and;

ii. Whether the students were able to access the sources of learning materials on their own while within the Moodle-based LMS. This was done by analyzing the number of objects (external learning sources or documents) accessed by the learners in the simulated e-learning system.

\subsection{Evaluation Results}

\section{a. Results from the survey}

The survey (on a sample size of 58) was carried out in comparison with the initial survey which informed the research the need for an SOA framework. The survey participants were also part of those who were involved in the survey that informed the research on the need for the SOA framework. The inspectors were first required to carry out the Abstract Task activities before responding to the questions raised in the survey. The analysis of collected data (using SPSS tool) indicated that there was an increment on the level of motivation on the learners as compared to the level of motivation as indicated on the survey carried out on the use of the pedagogical based eLearning system which the learners used initially.

Participants in the evaluation process could access a pre-set course in the Moodle course module. From the course the learners could then access the Wordpress Site within the course. The wordpress site provided a platform through which learners could collaborate and interact. There were other links that were provided within the course module which when clicked led to access of interactive course content/resources from remote websites. These sites exposed their content as web services which were then fetched while within the pre-set course in Moodle LMS. The SOA framework delivered a design model that incorporated the adult learners' characteristics, it allowed the adult learners to feel that the experience and intellectual ability were respected and appreciated.

It was observed that $76 \%$ of learners were in agreement that the SOA-based eLearning system provides more sources of learning materials while $58 \%$ indicated that they felt their experiences and previous achievements were acknowledged and respected. This is in concord with other previous studies [6] [8] [12] which established that when left to explore on their own, they feel respected, more satisfied and motivated to engage in e-learning and that adult learners like taking their own responsibility in their learning as they are independent and autonomous in their thinking [8][12]. From the survey it can be concluded that the SOA framework delivers an adult learning environment that utilizes the learner's capabilities, experiences and adult learning characteristics.

\section{b. Results from the system views/logs}

There were two types of system logs/views that were collected to establish the implementation of adult learners' experiences and self-directedness in the SOA framework respectively.

i. Measure for incorporation of adult learners' experiences in the creation of self-motivated learning materials

The system logs collected from the system were used to establish whether the students exchanges and access of remote objects/content increased positively to a point of demonstrating whether the students were able to collaborate and interact with one another within the wordpress blogging platform. The logs captured by the system indicated the number of participants who accessed the platform and either viewed other students' posts or created their own posts. There were a total of 22 students whose logs were recorded into the system and analysed as follows showing the students who accessed and viewed other students' posts. There was an 
average of 2.6 views for each student which indicates that approximately each student was able to view or interact with others at least 3 times.

The average number of exchanges shows that for each post created, there were 3 other posts/comments on the original posts created. This research study was able to observe that through the SOA framework, collaboration among instructor and learners was achieved as compared to the current elearning systems where there is no collaboration at all.

\section{ii. Measure for provision of self-directed learning}

The course that was created on the SOA framework for evaluation purpose provided access to 3 external education applications that delivered remote content in the Moodle based LMS as services. Since the total number of students who accessed the remote systems was 22 , the average number of access for each student to the external systems is 3 indicating that for each of the 3 educational applications each student had an access.

The system logs/views analysis shows that the collaborative and interactive learning environment which was made available for students allows the students to make use of their experiences to collaborate and interact among themselves.

\section{CONCLUSION}

Adults are independent, experienced and self-directed when it comes to learning. If they are treated the same way as children, they feel that their independence and experience in the knowledge gained is never appreciated or acknowledged [14].

This study established that there is a possibility of using an andragogy based eLearning framework that runs on an SOA platform. The SOA framework employs the Wordpress blogging tool in a Moodle LMS to facilitate collaboration as an important principle to adult learning process. The framework also allows access of external content which demonstrates collaboration at the institutional level and provides an environment for collaboration, creation of selfmotivated learning materials amongst adult learners. When adult learners, engaging in web-based e-learning are provided with a learning environment which allows them to collaborate interactively, they feel motivated to learn and this leads to their satisfaction [8] [12]. This way, adult learners' experiences are exploited and brought to the center of the learning process.

The SOA framework integrates the principles of adult learners into the learning process hence maintaining the motivation hence satisfaction for adult learners who carries their studies via e-learning environments. This is true when the adult learners' experiences are exploited and incorporated in the learning process and that provides them with opportunities to interact with themselves and instructors [6].

The SOA framework contributes to the improvement of the motivation of adult learners who sometimes may drop out of their learning because of lack of motivation as majority of the eLearning systems currently in use are more appropriate for younger learners. Knowles [13] [14] also argues that adults should not be treated the same way as children when it comes to learning.

However the SOA framework has a perceived weakness. The blogging feature, implemented by the framework, though widely adopted by many has never been used in a formal environment to provide learning process. Therefore its adoption into the LMS through the SOA framework will only be able to provide creation of self-motivated materials informally. As its adoption into the learning process now takes place, one feature lacks; how do we make the exchanges of ideas within the LMS formal and thereafter accept the selfmotivated material for use by others in future. The big question is; is it possible to make the Wordpress blogging functionality formal within an LMS and hence part of learning? If so, how will this be achieved especially through the use of the Service Oriented framework developed and implemented in this study?

\section{ACKNOWLEDGMENT}

The author(s) wish to acknowledge that this research study was supported by a Scholarship award from German Academic Exchange Service (DAAD).

\section{REFERENCES}

[1] Alonso, G. \& Casati, F., 2005. Web services and serviceoriented architectures. Data Engineering, 2005. ICDE 2005.

[2] Ardito, C., Costabile, M.F., Angeli, A., \& Lanzilotti, R. 2006. Systematic Evaluation of e-Learning Systems : an Experimental Validation. Proceedings of the 4th Nordic conference on Human-computer interaction: changing roles, pp.195-202.

[3] Bichelmeyer, B., 2005. Best Practices in Adult Education and E-Learning: Leverage Points for Quality and Impact of CLE. Val. UL Rev., 40(2), pp.509-520.

[4] Billington, D., 2000. Seven Characteristics of Highly Effective Adult learning Programs. The Adult Learner in Higher Education and the Workplace, pp.1-4.

[5] Cercone, K., 2008. Characteristics of Adult Learners with Implications for Online Learning Design. AACE Journal, 16, pp.137-159.

[6] Holton, D.L., 2010. Using Moodle to teach constructivist learning design skills to adult learners. In T. Kidd \& J. Keengwe, eds. Adult Learning in the Digital Age: Perspectives on Online Technologies and Outcomes. Information Science Reference, pp. 40-51.

[7] Holton, D.L., 2010. Using Moodle to Teach Constructivist Learning Design Skills to Adult Learners. , pp.1-12.

[8] Ismail, I., Gunasegaran, T. \& Idrus, R.M., 2010. Does Elearning Portal Add Value to Adult Learners? , 2(5), pp.276-281.

[9] Johnson, D., 2010. Teaching with author's blogs: Connection, Collaboration \& Creativity. Journal of Adolescent \& Adult Literacy, 53(3), pp.172-180.

[10] Jun, J., 2005. Understanding dropout of adult learners in e-learning. , (1). Available at: https://getd.libs.uga.edu/pdfs/jun_jusung_200505_phd.pd f [Accessed August 27, 2014]. 
[11] Jun, J., 2004. Understanding the factors of adult learners dropping out of e-learning courses. The $45^{\text {th }}$ annual Adult Education Research Conference, pp.279-284.

[12] Kim, K. J., 2009. Motivational challenges of adult learners in self-directed e-learning. Journal of Interactive Learning Research, 20(3), pp.317-335.

[13] Knowles, M., 1996. The ASTD training \& development handbook: A guide to human resource development 4th ed. R. Craig, ed., New York: McGraw-Hill.

[14] Knowles, M., 1980. The modern practice of adult education, New York: Association Press, \& Cambridge Book Publishers.

[15] Leal, J. \& Queirós, R., 2011. Using the Learning Tools Interoperability Framework for LMS Integration in Service Oriented Architectures. Technology Enhanced Learning.

[16] Luthria, H. \& Rabhi, F., 2009. Service Oriented Computing in Practice: An Agenda for Research into the Factors Influencing the Organizational Adoption of Service Oriented Architectures. Journal of theoretical and applied electronic commerce research, 4(1), pp.39-56.

[17] MacLennan, E. \& Van Belle, J.P., 2014. Factors affecting the organizational adoption of service-oriented architecture (SOA). Information Systems and e-Business Management, 12, pp.71-100.

[18] Papazoglou, M.P. et al., 2008. Service-Oriented Computing: A Research Roadmap. International Journal of Cooperative Information Systems, 17, pp.223-255.

[19] Rodrigues, A.A., 2012. Empowering Adult Learners through Blogging with iPads and iPods.

[20] Eduardo M. D. Marques and Paulo N. M. Sampaio, 2012. "NSDL: An Integration Framework for the Network Modeling and Simulation," International Journal of Modeling and Optimization vol. 2, no. 3, pp. 304-308

[21] Open Knowledge Initiative (O.K.I.) Website. http://www.okiproject.org.

[22] IMS Digital Repositories Interoperability - Core Functions Information Model Revision: 13 January 2003 